\title{
Measuring human-error probabilities in drug preparation: a pilot simulation study
}

\author{
P. Garnerin • B. Pellet-Meier • P. Chopard • \\ T. Perneger $\cdot$ P. Bonnabry
}

Received: 16 November 2006 / Accepted: 26 April 2007 / Published online: 31 May 2007

(C) Springer-Verlag 2007

\begin{abstract}
Objectives Designing a safe medication process requires the ability to model its reliability using methods such as probabilistic risk assessment (PRA). However, lack of data, especially on human-error probabilities (HEPs), limits its use. To assess whether small-scale simulations could help generate HEP data, a pilot study was conducted among nurses and anaesthetists. It focused on two core activities, namely, the manual preparation of medications and the arithmetic necessary to prepare drugs. Its specific objectives were to evaluate whether HEPs could be high enough to be measurable and to determine whether these HEPs could be sensitive to individuals and task details. These would give some insight into the level of detail required by PRA analysis.

Methods Thirty nurse and 28 anaesthetist volunteers were involved in the experiment. Nurses and anaesthetists had to prepare medications for 20 patients and 22 syringes of various drugs, respectively. Both groups had to perform 22 calculations relating to the preparation of drugs. HEPs, distribution of HEPs and dependency of HEPs on individuals and task details were assessed.
\end{abstract}

P. Garnerin $(\bowtie)$

Anaesthesiology Service, Geneva University Hospitals,

Rue Micheli-du-Crest, 24,

CH-1211 Geneva 14, Switzerland

e-mail: philippe.garnerin@hcuge.ch

B. Pellet-Meier • P. Bonnabry

Hospital Pharmacy, Geneva University Hospitals,

Geneva, Switzerland

P. Chopard · T. Perneger

Quality of Care Service, Geneva University Hospitals,

Geneva, Switzerland
Results In the preparation tasks, overall HEP was 3.0\% for nurses and $6.5 \%$ for anaesthetists. In the arithmetic tasks, overall HEP was $23.8 \%$ for nurses and $8.9 \%$ for anaesthetists. A statistically significant difference was noted between the two groups. In both preparation and arithmetic tasks, HEPs were dependent on individual nurses but not on individual anaesthetists. In every instance, HEPs were dependent on task details.

Conclusion Our study illustrates that small-scale simulations represent an interesting way of generating HEPs. HEPs are, indeed, in the range of $10^{-2}$ and $10^{-1}$. But in most cases, HEPs depend heavily on operators and task details. This dependency means that the influence of these parameters must be determined before advanced PRA analysis. There is therefore an urgent need to develop experimental research into assessing this influence by means of randomised controlled trials.

Keywords Drug-delivery system · Medical errors ·

Safety management

\section{Introduction}

The well-publicised report of the Institute of Medicine, To Err is Human [1], recommends using the qualitative and quantitative methods developed for high-risk industries [2] to prevent errors in the medication process, a leading cause of avoidable deaths [1]. Because they are easy to use, qualitative tools are gaining popularity among specialists of medication safety. Root-cause analysis [3], for example, reveals the organisational factors that contributed to adverse drug events [4], whereas failure mode, effects and criticality analysis $[5,6]$ prospectively helps to rank the potential hazards of the medication process [7, 8]. However, as with 
many care processes, the medication process comprises a complex sequence of activities, during which many human errors (e.g. errors in dose prescription or drug selection) can take place and combine in multiple ways, leading to an adverse event. If safety is to be obtained by design, it becomes essential to be able to model such complex causation mechanisms and to measure, a priori, the failure rate of the medication process. Indeed, modelling provides a way of investigating the various possible configurations so as to identify options having an acceptable risk-benefit ratio. In that respect, probabilistic risk assessment (PRA) represents a powerful prospective tool. Using "AND" and "OR" logical gates, a medication error can be modelled as a combination of elementary human errors (i.e. errors that represent "basic events which cannot be subdivided" [9]), such as error in selecting a drug or labelling a syringe, that are then assembled into an event tree materialising the various ways in which the process can fail. Furthermore, knowing the probability of each elementary human error [human-error probabilities (HEP)] makes it possible to compute the failure rate of the process (Fig. 1).

Although suggestions to apply PRA to patient-safety issues have recently been made $[10,11]$, its use remains embryonic in health care. One reason for its slow dissemination is that it relies on data that are seldom found in medical literature. For instance, analysing a medication process using PRA would at least require descriptions of the different tasks performed and of the possible alternative tasks. For each of them, a detailed inventory of the elementary human errors that could occur, including the mean and distribution of corresponding HEPs, should be obtained [12]. In addition, independence of HEPs from individuals and task details such as the drug on hand, its packaging or the type of calculation (e.g. flow, conversion of units) should be assessed. Indeed, constant HEPs are not only a prerequisite for PRA analysis [13] but also for developing reliable processes [14].

With regard to the medication process, a first potential source of data suitable for the generation of HEPs is represented by the many studies that document operating experience [15]. Unfortunately, HEP data are difficult to extract from these studies. Indeed, reported error rates often relate to an entire process or to some of its segments (e.g. prescribing, dispensing) but not to the different tasks it includes. For example, a rate of wrong-dose error-defined as "when the patient receives an amount of medicine that is greater than or less than the amount ordered" [16] - is impossible to convert into HEPs respectively associated with selecting the wrong concentration of a drug, failing to dilute it, or injecting the incorrect volume. Moreover, how the distribution of these HEPs is influenced by factors such as individuals or task details remains unknown.

A second potential source of data could be experimental research. By having individuals repeat the same tasks under identical circumstances, experimental research allows a more precise investigation into how HEPs are influenced by operators, task details or performance-shaping factors such as fatigue or time pressure [12]. Moreover, by providing an environment in which the nature of the various tasks can be altered and controlled, it affords a way of improving human reliability at reasonable cost by testing alternatives before implementation.
Fig. 1 Simplistic event-tree model of a medication error. The model was built for three prepared syringes presented together on a tray. The pathway from various elementary human errors (circle) to a medication error is represented as an event tree using AND and OR logical gates. The medication error occurs either because one of the syringes contains the wrong drug or concentration or because of a syringe swap.

Corresponding human-error probabilities (HEP) (bold) have been attributed to each elementary human error and combined to compute the probability of medication error. Introducing ready-to-use products prepared by pharmaceutical companies should eliminate dilution errors and reduce the probability of a medication error (italics)

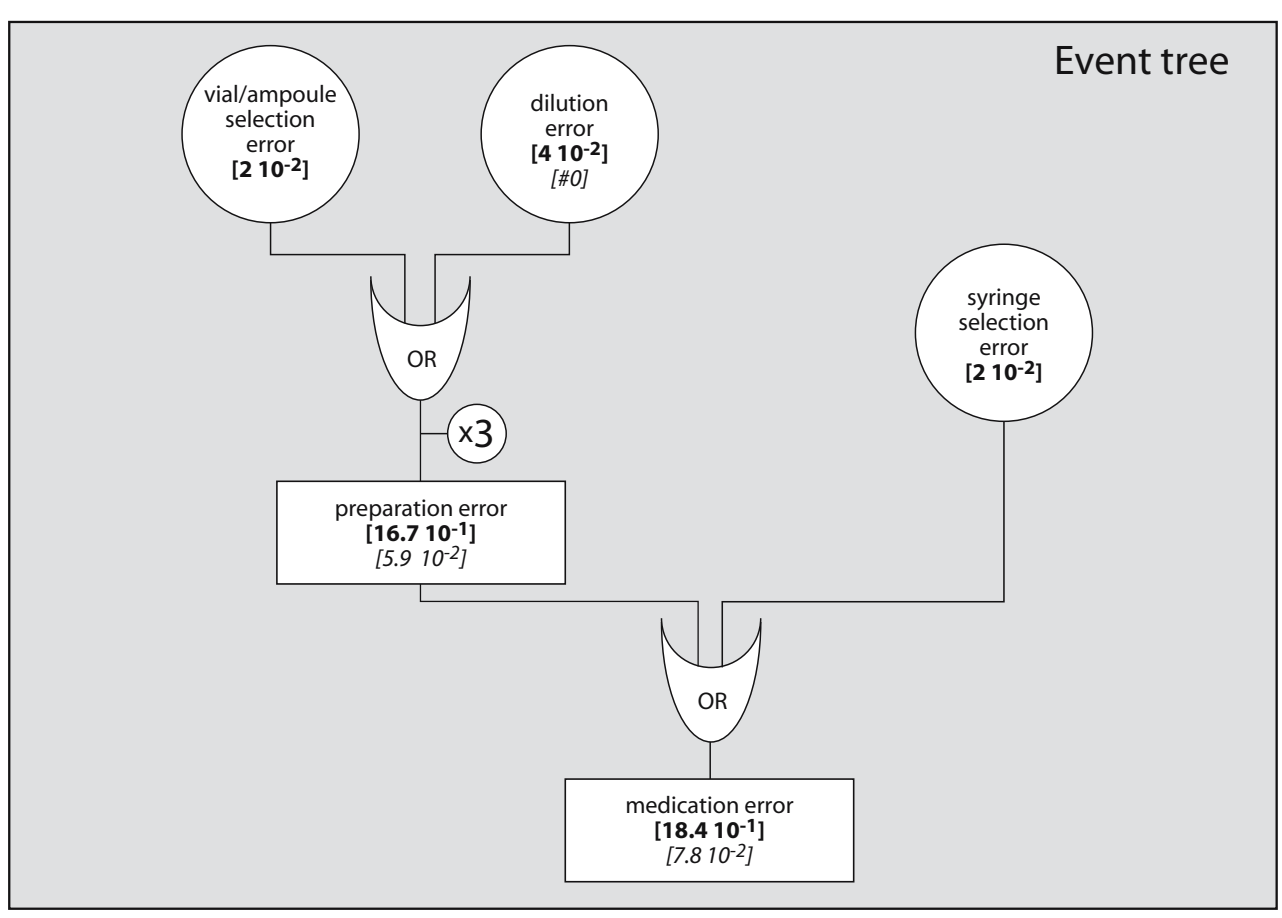


Before developing dedicated experiments to investigate specific human errors and applying PRA to the medication process, we conducted a pilot study. Its aim was to evaluate the potential for generating HEP data of small-scale simulations involving a limited number of volunteers. To apprehend a large spectrum of circumstances, we decided to simulate two core activities of an actual medication process, namely, the manual preparation of medications and the arithmetic necessary to prepare drugs. The study, which incorporated a variety of realistic task details, was, in addition, conducted in two distinct groups of professionals: nurses and anaesthetists. Its specific objectives were first to evaluate whether HEPs could be high enough to be measurable and then to assess whether these HEPs could be sensitive to operators and task details so as to get some insight into the level of detail required by PRA analysis.

\section{Materials and methods}

Three tasks were analysed in three different experiments conducted in a dedicated room of a university-affiliated tertiary hospital. In the first, nurses apportioned oral medications; in the second, anaesthetists prepared intravenous medications; in the third, both groups performed the arithmetic needed for preparing treatment and drugs. For each experiment, the task was repeated, its details being specified each time by an instruction. Each operator received the same set of instructions presented in the same order. For each of the three tasks, specific elementary human errors were identified.

\section{Study population}

Two volunteer groups of 30 nurses and 28 anaesthetists were involved in the study. The protocol received the approval of the hospital's research ethics committee. The volunteers, who were recruited in the institution by an advertising campaign, were informed about the aim and design of the study, gave their written consent, and received a $€ 35$ remuneration.

\section{Nurse preparation task}

Each nurse was asked to prepare 80 oral medications for the treatment of 20 patients for $24 \mathrm{~h}$. Each patient was prescribed four drugs up to four times a day. Orders, medications, equipment and preparation methods were similar to those usually used on wards, but transcribed orders were printed to avoid preparation errors being influenced by misread handwriting. The order for each patient had four instructions, one for each drug, and was attached to a medicine box with four compartments, one for each time of distribution $(08: 00,12: 00,18: 00,22: 00)$, into which the tablets had to be placed. Only common drugs in their original packaging that could be clearly identified in their medicine boxes after preparation were chosen. The drugs had to be taken from a small medication cabinet similar to those available on wards (Fig. 2). The cabinet contained 138 different drugs. To imitate real-life conditions, drugs were arranged in alphabetical order, and two inconsistencies were introduced: a box of Madopar 250 (levodopa $200 \mathrm{mg}+$ benserazide $50 \mathrm{mg}$ ) was placed behind a box of Madopar 250 DR (dual-release form), and one of the two blisters in a box of dexamethazone $4 \mathrm{mg}$ was replaced by a similar blister of phenobarbital $100 \mathrm{mg}$. The cabinet was identically set up for all participants.

After the nurse had prepared all the medications, a pharmacist compared them twice with corresponding controls. Preparation errors [16] were subdivided into four distinct elementary human errors categorised as commission or omission errors. Commission errors included selection, counting or scheduling errors. A selection error was registered when either the wrong medication was chosen or the prescribed medication was presented in the wrong dosage or form (e.g. immediate vs. slow release). A counting error was noted when, in the absence of a selection error, the number of tablets in a medicine box was different from the expected number. A scheduling error was identified when in the absence of a count error, tablets were found in the wrong compartment (e.g. for 08:00 instead of for 12:00). When both the selected dosage and the number of tablets in a compartment differed from the prescription, no error was counted if the total amount of drug equalled the prescribed dose. Unless the deviation had already been categorised as commission error, an omission error was recorded each time a prescribed drug was not found in the medicine box.

\section{Anaesthetist preparation task}

Each anaesthetist was asked to prepare 22 syringes using ten trays, with each tray containing five to six drugs and ten to 16 ampoules. Overall, 14 different drugs corresponding
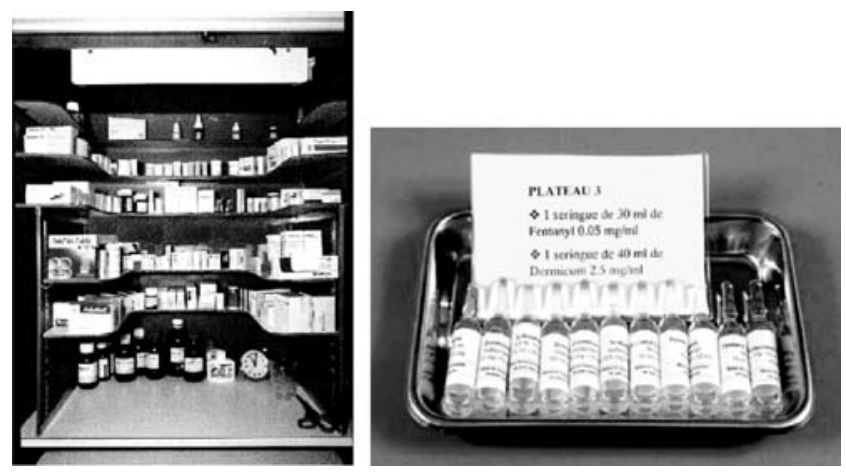

Fig. 2 Standardised medication cabinet and tray used in the experiment 
to 28 products varying in concentration and volume were used. A printed order containing instructions, one for each syringe, detailing which medication had to be prepared in terms of drug, concentration and volume was joined to each tray (Fig. 2). To minimise the cost of the experiment, ampoules filled with water were prepared and labelled by the hospital pharmacy. Labels displayed information similar to that for the real products. No specific means were used to make the drugs easier to distinguish by sight. A vial of sodium chloride, syringes and needles were provided for drug dilution. While preparing drugs, anaesthetists were observed by a pharmacist who also checked the volumes in the remaining ampoules. Preparation errors were again subdivided into four distinct elementary human errors categorised as commission or omission errors. Commission errors included errors in selection, dilution or volume. A selection error was noted when a medication departing from the one prescribed was chosen. In the absence of selection error, a dilution error was counted when the final concentration did not correspond to the one prescribed. Finally, in the absence of concentration error, a volume error was recorded if the final volume did not equal the required volume. An omission error was documented each time a syringe was not prepared.

\section{Arithmetic task}

Each nurse and anaesthetist was asked to perform 22 calculations (instructions) relating to the preparation of drugs, such as determining dilution or flow, splitting doses over $24 \mathrm{~h}$, or converting units. Calculations were easy to perform mentally and were typical for medical and surgical wards. Examples included: "how many $\mathrm{ml}$ represent $10 \mathrm{mg}$ of morphine when morphine solution $0.1 \%$ is used?" or, "if a patient is prescribed 600,000 international units (IU) of Mycostatin, three times a day, what is the length of the treatment that can be conducted with three vials of $24 \mathrm{ml}$, $100,000 \mathrm{IU} / \mathrm{ml}$ ?" No calculator was allowed. Any wrong answer was counted as an elementary human error.

\section{Statistical analysis}

For each of the three tasks, an overall HEP as well as HEPs per type of elementary human error were calculated by dividing the number of errors observed by the number of opportunities for error (i.e. the number of instructions) [12]. In addition, regular and cumulative statistical distributions of overall HEPs were established for both operators and task details (instructions). The hypothesis that HEPs were constant was assessed using Fisher's exact test. Nurses' and anaesthetists' HEP distributions for the arithmetic task were compared using the Mann-Whitney test. A $p$ value lower than 0.05 was considered significant. Statistical analyses were done using SPSS 11.0.

\section{Results}

For the nurse preparation task, the 30 operators committed 71 errors in a total of 2,400 instructions for an overall HEP of $3.0 \%$. HEPs ranged from $0 \%$ to $8.8 \%$ for operators and from $0 \%$ to $63.3 \%$ for instructions (Fig. 3). The most frequent errors were selection errors, where the HEP was $2.1 \%$ (Table 1). For instructions, the two highest HEPs (63.3\% and $40.0 \%$, respectively) were associated with the two deliberate anomalies introduced for the sake of realism. Other increased HEPs were linked to products with multiple dosage forms or strengths and to tablets that had to be cut (Table 2).

For the anaesthetist preparation task, the 28 operators committed 40 errors in a total of 616 instructions. Thus, the overall HEP was $6.5 \%$. HEPs ranged from $0 \%$ to $18.2 \%$ for operators and from $0 \%$ to $46.4 \%$ for instructions (Fig. 3). The most frequent errors were dilution errors, where the HEP was $3.4 \%$ (Table 1). For instructions, the highest HEP (46.4\%) was linked to confusion over the volume of an ampoule, probably because the volume information was not noticed, being printed on the same line as the concentration details. The next highest HEP (14.3\%) was linked to mistakes in calculating the required volume from a concentration expressed in percent (Table 2). Deviations from expected concentration ranged from ten times lower to two times higher.

For the arithmetic task, nurses and anaesthetists committed 157 and 55 errors in a total of 660 and 616 instructions, respectively. The corresponding HEPs were $23.8 \%$ for nurses, $8.9 \%$ for anaesthetists and $16.6 \%$ overall. Among nurses, HEPs ranged from $0 \%$ to $72.7 \%$ for operators and from $0 \%$ to $60.0 \%$ for instructions. Among anaesthetists, HEPs ranged from $0 \%$ to $27.3 \%$ for operators and from $0 \%$ to $75.0 \%$ for instructions (Fig. 3). A statistically significant difference was noted between nurses and anaesthetists $(p<0.0001)$. With regard to instructions, the highest HEP (67.2\%) was associated,- - among both nurses and anaesthetists - to an instruction assuming the knowledge that an open vial could not be kept for future injections. The question "how many bottles of Metronidazole $500 \mathrm{mg} 500 \mathrm{ml}$ are required to administer a 5-day intravenous treatment to a patient of $75 \mathrm{~kg}$ using a first dose of $15 \mathrm{mg} / \mathrm{kg}$ and following doses of $7.5 \mathrm{mg} / \mathrm{kg}$ every 6 hours?" had consequently a corresponding HEP of $60.0 \%$ for nurses and $75.0 \%$ for anaesthetists. The second highest HEP (29.3\%) was linked to the use of micrograms. The question "with an ampoule of Neupogen (filgrastim) $1 \mathrm{ml}$ $30 \mu \mathrm{g} / \mathrm{ml}$, how much glucose G5 is required to obtain a final concentration of $1.5 \mu \mathrm{g} / \mathrm{ml}$ ?" had an associated HEP of $53.3 \%$ for nurses but only $3.6 \%$ for anaesthetists.

In both preparation and arithmetic tasks, HEPs were dependent on individual nurses but appeared to be constant 
nurse preparation task ( 30 nurses, 80 instructions)

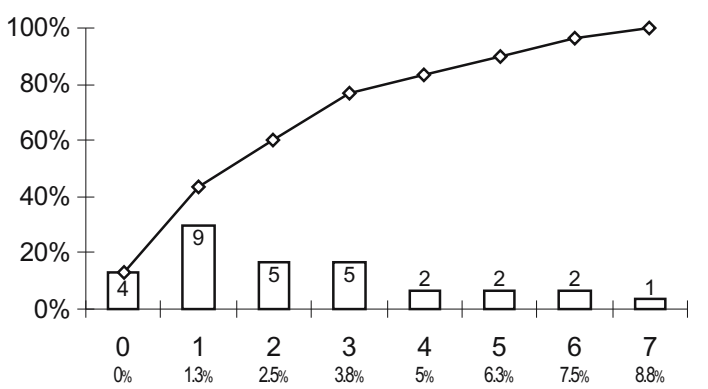

anaesthetist preparation task (28 anaesthetists; 22 instructions)

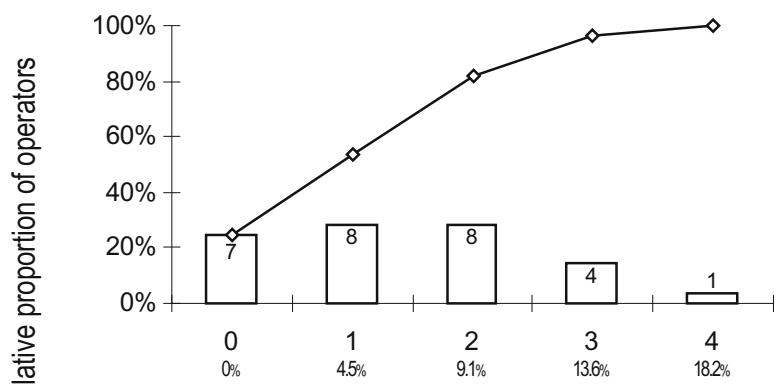

nurse calculation task ( 30 nurses; 22 instructions)

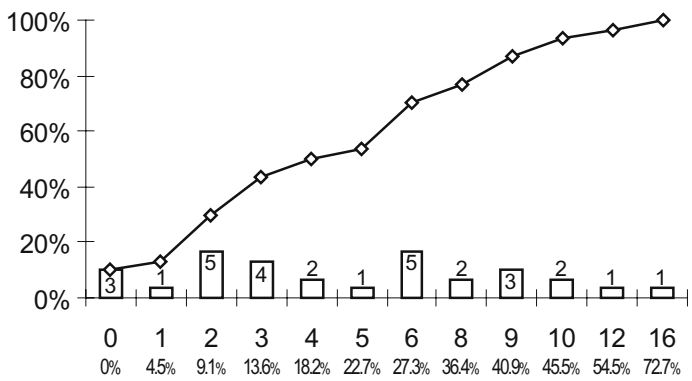

anaesthetist calculation task (28 anaesthetists; 22 instructions)

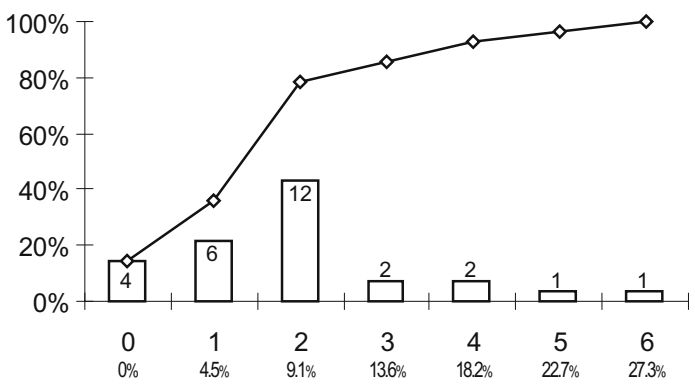

number of errors and HEP per operator

Fig. 3 Distribution of errors. For each task (horizontal strip), the columns show the graphs and figures for individuals on the left and for instructions on the right. Example of a reading: for the anaesthetist preparation task (second strip), two errors (left graph) were made by eight individuals (28.6\% of individuals) for a human-error probability (HEP) of $9.1 \%$ (two
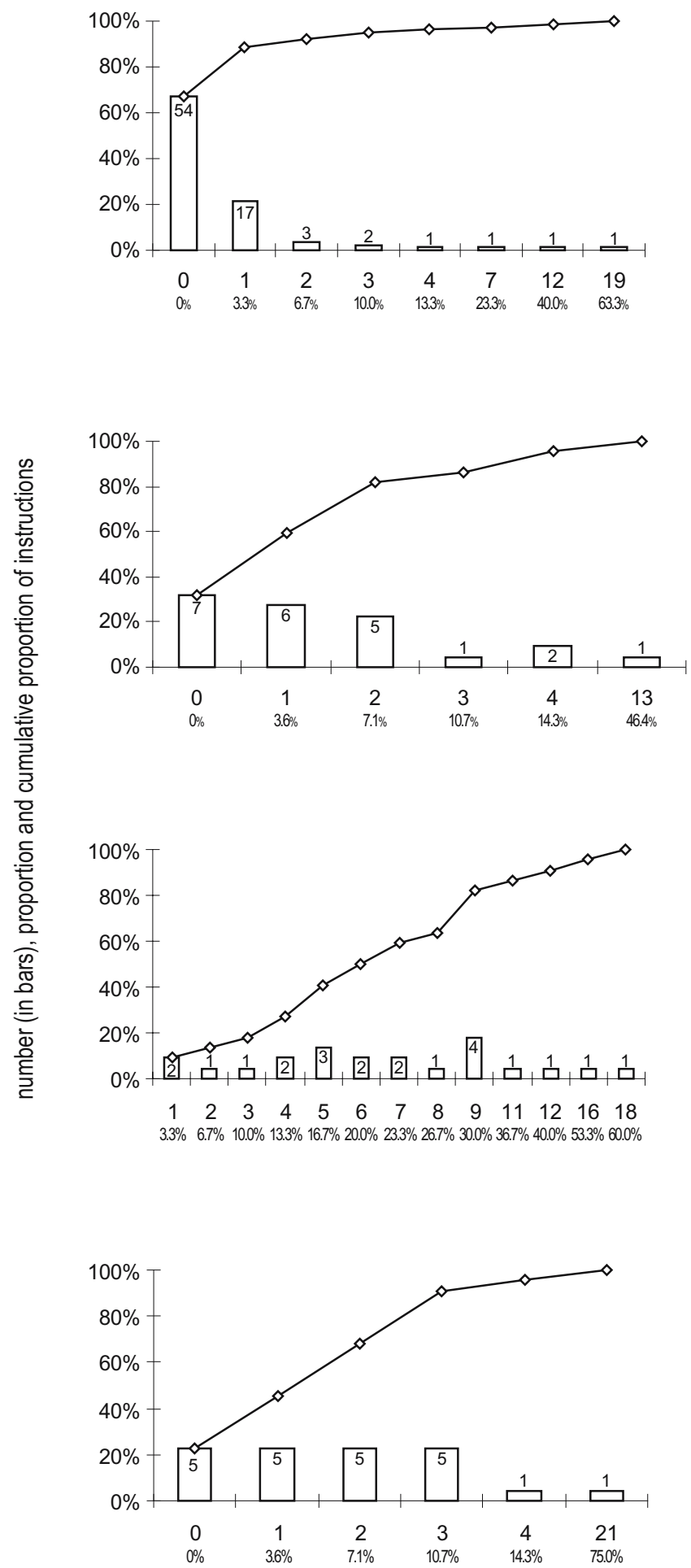

number of errors and HEP per instruction

errors in 22 tasks). Overall, $82.1 \%$ of individuals made two or fewer errors, i.e. had a HEP equal to or lower than $9.1 \%$. One error (right graph) was made in six instructions (27.3\% of instructions), for a HEP of $3.6 \%$ (one error for 28 individuals). Overall, 59.1\% of instructions generated one or fewer errors, i.e. had a HEP equal to or lower than $3.6 \%$ 
Table 1 Nurse and anaesthetist preparation experiments: number of errors and human-error probability (HEP)

\begin{tabular}{lllllll}
\hline & All errors & \multirow{2}{*}{ Omission errors } & \multicolumn{2}{l}{ Commission errors } & \\
\cline { 3 - 6 } & & & Selection error & Count/volume error & Dilution error & Scheduling error \\
\hline Nurses $(n=2400)$ & $71-3.0 \%$ & $5-0.2 \%$ & $51-2.1 \%$ & $10-0.4 \%$ & NA & $5-0.2 \%$ \\
Anaesthetists $(n=616)$ & $40-6.5 \%$ & $6-1.0 \%$ & $11-1.8 \%$ & $2-0.3 \%$ & $21-3.4 \%$ & NA \\
\hline
\end{tabular}

$N A$ not applicable

for anaesthetists, who can therefore be considered equally prone to errors. In all three tasks, HEPs were dependent on the details of each task (Table 3 ).

\section{Discussion}

Our findings demonstrate that for various activities and professional groups, HEPs can be measured by means of small-scale simulations. These probabilities are in accordance with those obtained by analysing operating experience, and they add to the growing body of evidence that for the preparation of drugs, most HEPs are in the range of $10^{-2}$ to $10^{-1}$. Indeed, our results are consistent with previously published error rates, which-when obtained with comparable methodologies - can be converted to
HEPs. The 3.0\% HEP observed for the nurse preparation task is of the same order of magnitude as the $2.1 \%$ [17] and $1.7 \%$ [18] figures found in two observational studies of pharmacists or pharmacy technicians involved in the manual dispensing of drugs. Likewise, the 6.5\% HEP measured for the anaesthetist preparation experiment is comparable with the $11.6 \%$ error rate revealed, for both one-step and multiple-step preparations, by an observational study on intravenous drug errors [19]. With regard to the arithmetic task, the $23.8 \%$ HEP observed for nurses in our study is close to the $25 \%$ figure measured during a 20 -item arithmetic test completed by nurses under experimental conditions [20].

Our results also give an insight into the magnitude of variations in HEPs in relation to task details such as drugs

Table 2 Most common preparation errors

\begin{tabular}{|c|c|c|c|c|}
\hline Instruction & $\begin{array}{l}\text { Number } \\
\text { of errors }\end{array}$ & HEP & Type of error & Nature of the error \\
\hline \multicolumn{5}{|l|}{ Nurses $(n=30)$} \\
\hline Madopar 250 [12 h: 1; 20 h: 1] & 19 & $63.3 \%$ & Selection errors & Madopar 250 DR used instead \\
\hline Dexamethazone $4 \mathrm{mg}$ [8 h: 1$]$ & 12 & $40.0 \%$ & Selection errors & Phenobarbital $100 \mathrm{mg}$ used instead \\
\hline $\begin{array}{l}\text { Co-Dafalgan effervescent [ } 8 \mathrm{h:} 1 ; 16 \mathrm{h:}: 1 ; 20 \mathrm{h:} \text { 1] } \\
\text { (acetaminophen } 500 \mathrm{mg}+\text { codeine } 30 \mathrm{mg} \text { ) }\end{array}$ & 7 & $23.3 \%$ & Selection errors & Regular Co-Dafalgan used instead \\
\hline Tranxilium $10 \mathrm{mg}[8 \mathrm{h:} 1 ; 20 \mathrm{h:} 1]$ & 4 & $13.3 \%$ & $\begin{array}{l}1 \text { selection error; } 1 \\
\text { count error; } 2 \\
\text { omission errors }\end{array}$ & $\begin{array}{l}\text { Tranxilium } 5 \text { mg used instead; } 1 \text { tablet } \\
\text { missing }\end{array}$ \\
\hline Depakine chrono $250 \mathrm{mg}$ [8 h: 1] (valproic acid) & 3 & $10.0 \%$ & Selection errors & $\begin{array}{l}\text { Depakine chrono } 500 \mathrm{mg} \text { only available; } \\
\text { tablets not cut }\end{array}$ \\
\hline $\begin{array}{l}\text { Isoket retard } 20 \mathrm{mg} \text { [8 h: } 1 ; 12 \mathrm{~h}: 1 ; 20 \mathrm{~h}: 1] \\
\text { (isosorbide dinitrate-delayed action) }\end{array}$ & 3 & $10.0 \%$ & Selection errors & Regular Isoket $20 \mathrm{mg}$ used instead \\
\hline \multicolumn{5}{|l|}{ Anaesthetists $(n=28)$} \\
\hline \multirow[t]{2}{*}{ Nubain (nalbuphine) $2 \mathrm{mg} / \mathrm{ml} ; 1$ syringe of $10 \mathrm{ml}$} & 13 & $46.4 \%$ & 12 dilution errors & $\begin{array}{l}2 \text { ampoules of Nubain } 10 \mathrm{mg} / \mathrm{ml} 2 \mathrm{ml} \\
\text { used instead of } 1\end{array}$ \\
\hline & & & 1 selection error & $\begin{array}{l}1 \text { ampoule of Nubain } 10 \mathrm{mg} / \mathrm{ml} 2 \mathrm{ml} \text { and } \\
1 \text { ampoule of ephedrine } 10 \mathrm{mg} / \mathrm{ml} 2 \mathrm{ml} \\
\text { used }\end{array}$ \\
\hline Lidocaine $7.5 \mathrm{mg} / \mathrm{ml} ; 1$ syringe of $40 \mathrm{ml}$ & 4 & $14.3 \%$ & Dilution errors & $\begin{array}{l}\text { Wrong amount of product withdrawn } \\
\text { from ampoules of lidocaine } 1 \% 10 \mathrm{ml}\end{array}$ \\
\hline Xylocaine $0.25 \% ; 2$ syringes of $40 \mathrm{ml}$ & 4 & $14.3 \%$ & Omission errors & 1 syringe not prepared \\
\hline Carbostesin-adrenaline $0.5 \% ; 1$ syringe of $20 \mathrm{ml}$ & 3 & $10.7 \%$ & Selection errors & $\begin{array}{l}\text { Ampoules of Carbostesin } 0.5 \% 5 \mathrm{ml} \\
\text { used instead of Carbostesin-adrenaline } \\
0.5 \% 5 \mathrm{ml}\end{array}$ \\
\hline
\end{tabular}

HEP human-error probability 
Table 3 Independence of human-error probabilities (HEPs) from individuals and instructions

\begin{tabular}{|c|c|c|c|c|c|c|}
\hline & & \multicolumn{2}{|c|}{ Preparation experiment } & \multicolumn{3}{|c|}{ Arithmetic experiment } \\
\hline & & Nurse & Anaesthetist & Overall & Nurse & Anaesthetist \\
\hline \multirow[t]{5}{*}{ Individuals } & HEP & $3.0 \%$ & $6.5 \%$ & $16.6 \%$ & $23.8 \%$ & $8.9 \%$ \\
\hline & Number of instructions per individual & 80 & 22 & 22 & 22 & 22 \\
\hline & Number of individuals per instruction & 30 & 28 & 58 & 30 & 28 \\
\hline & Independence (constant) & no & yes & no & no & yes \\
\hline & $P$ value & 0.02 & 0.5 & $<10^{-4}$ & $<10^{-4}$ & 0.3 \\
\hline \multirow[t]{2}{*}{ Instructions } & Independence (constant) & no & no & no & no & no \\
\hline & $P$ value & $<10^{-4}$ & $<10^{-4}$ & $<10^{-4}$ & $<10^{-4}$ & $<10^{-4}$ \\
\hline
\end{tabular}

that look or sound alike, label legibility or use of unfamiliar units (e.g. \%, $\mu$ g). Numerous case reports [21] made such variations expected. For instance, the HEP associated with selection errors was 30 times higher than average when one of the two blisters in a box of Dexamethazone $4 \mathrm{mg}$ was replaced by a similar blister of Phenobarbital $100 \mathrm{mg}$; the HEP associated with dilution errors was 14 times its average when a $10-\mathrm{ml}$ syringe of nalbuphine $2 \mathrm{mg} / \mathrm{ml}$ had to be prepared from 2-ml ampoules of nalbuphine $10 \mathrm{mg} /$ $\mathrm{ml}$. Similar findings with regard to operators emerged from our study, as strong differences in HEPs were observed within the group of nurses and between the two groups of professionals. For example, in one nurse, the HEP corresponding to calculation errors was three times higher than its average; this HEP was also averaged three times higher in nurses than in anaesthesiologists.

High HEPs and large variations in HEPs both for operators and task details such as those revealed by our study signal flawed processes [14] and would require an immediate remedy in industry [22]. However, our results suggest that prior to designing reliable medication processes using PRA analysis, two entangled methodological limitations have to be overcome. First, elementary human errors have to be defined at the appropriate level. Indeed, when HEPs are not independent from task details, as observed in our study, tasks can no longer be considered equivalent and must be categorised. For example, if the HEP associated to selection errors depends on whether one or several formulations of a drug are available in the medication cabinet, then two distinct tasks have to be incorporated into PRA analysis (i.e. the event tree). Consequently, the proportion of each task as well as corresponding HEPs should be measured. To make the problem worse, many more categories of potentially nonequivalent tasks could be obtained by combining specific tasks details. For instance, the HEP associated with dilution error could simultaneously depend upon both the type and size of the font used on labels and the concentration format ( $\mathrm{mg} / \mathrm{ml} \mathrm{vs}$. \%). This necessary but potentially endless refinement of PRA analysis uncovers the second methodological limitation, namely, the lack of appropriate data.
One must indeed recognise that the analysis of operating experience so far has yielded very little information about the various factors that make a medication process hazardous or safe [23]. The extent of variations in HEPs revealed by our results requires a move from traditional observational studies towards experimental research based on randomised controlled trials. In this regard, much remains to be done, although some task details [24-27] as well as a few performance-shaping factors are already under investigation [28-30]. For instance, the reliability of various tasks in relation to label design (e.g. ways of expressing drug dosage), medication cabinets, trolleys or trays and the use of information technologies [31] could be explored. With regard to operators, methods for improving arithmetic skills could be tested [20], and professionals could be licensed and relicensed [32] to carry out specific tasks within the medication process. This would help implement the principle of equivalent operators, one of the pillars of safe systems as put forward by Amalberti et al. [33].

\section{Conclusion}

Despite obvious obstacles, it is now time to follow humanfactor specialists by working out how tasks should be designed and assembled to create an efficient and secure medication process. In this regard, our study illustrates that small-scale simulations represent a promising way of generating HEPs suitable for PRA analysis. However, it also reveals the complexity of the approach and underlines the need for experimental research to accurately analyse how HEPs are influenced by individuals, task details and performance-shaping factors and to find ways of limiting their impact.

Acknowledgements The study was supported by the Quality of Care programme of the Geneva University Hospitals. It was done independently of this funding programme. The authors are indebted to Charles Vincent and Sally Adams for their enlightened and constructive discussion of the manuscript. 


\section{References}

1. Kohn L, Corrigan J, Donaldson M (2000) To err is human. Building a safer health system. National Academy Press, Washington, DC

2. Spath P (2000) Error reduction in health care. A systems approach to improving patient safety. Jossey-Bass Publishers, San Francisco

3. Vincent C, Taylor-Adams S, Chapman E, Prior S, Strange P, Tizzard A (2000) How to investigate and analyse clinical incidents: clinical risk unit and association of litigation and risk management protocol. BMJ 320:777-781

4. Rex JH, Turnbull JE, Allen SJ, Van de Voorde K, Luther K (2000) Systematic root cause analysis of adverse drug events in a tertiary referral hospital. Jt Comm J Qual Improv 26:563-575

5. Cohen M, Senders J, Davis N (1994) Failure mode and effects analysis: a novel approach to avoiding dangerous medication errors and accidents. Hosp Pharm 29:319-330

6. DeRosier J, Stalhandske E, Bagian J, Nudell T (2002) Using health care Failure Mode and Effect Analysis: the VA National Center for Patient Safety's prospective risk analysis system. Jt Comm J Qual Improv 28:248-267

7. Kunac DL, Reith DM (2005) Identification of priorities for medication safety in neonatal intensive care. Drug Saf 28:251-261

8. Bonnabry P, Cingria L, Sadeghipour F, Ing H, Fonzo-Christe C, Pfister RE (2005) Use of a systematic risk analysis method to improve safety in the production of paediatric parenteral nutrition solutions. Qual Saf Health Care 14:93-98

9. International Electrotechnical Commission. Fault tree analysis (FTA)-IEC 1025 (1990) Geneva: International Electrotechnical Commission.

10. Marx DA, Slonim AD (2003) Assessing patient safety risk before the injury occurs: an introduction to sociotechnical probabilistic risk modelling in health care. Qual Saf Health Care 12(Suppl 2):ii33-ii38

11. Wreathall J, Nemeth C (2004) Assessing risk: the role of probabilistic risk assessment (PRA) in patient safety improvement. Qual Saf Health Care 13:206-212

12. Taylor-Adams S, Kirwan B (1995) Human reliability data requirements. International Journal of Quality \& Reliability Management 12:24-46

13. Atwood C, LaChance J, Martz H, Anderson D, Englehardt M, Whitehead D, Wheeler T (2003) NUREG/CR-6823, Handbook of parameter estimation for probabilistic risk assessment. US Nuclear Regulatory Commission, Washington, DC

14. Berwick D (1991) Controlling variation in health care: a consultation from Walter Shewhart. Med Care 29:1212-1225

15. Allan Flynn E, Barker K (1999) Medication errors research. In: M. Cohen (eds) Medication errors. Jones and Bartlett, Sudbury pp $6.1-6.30$
16. Allan E, Barker K (1990) Fundamentals of medication error research. Am J Hosp Pharm 47:555-571

17. Hassall T, Daniels C (1983) Evaluation of three types of control chart methods in unit dose error monitoring. Am J Hosp Pharm 40:970-975

18. Taylor J, Gaucher M (1986) Medication selection errors made by pharmacy technicians in filling unit dose orders. Can J Hosp Pharm 39:9-12

19. Taxis K, Barber N (2003) Ethnographic study of incidence and severity of intravenous drug errors. BMJ 326:684-687

20. Bayne T, Bindler R (1997) Effectiveness of medication calculation enhancement methods with nurses. J Nurs Staff Dev 13:293-301

21. Cohen M (1999) Medication errors: causes, prevention, and risk management. John and Bartlett, Sudbury

22. Leape L (1994) Error in medicine. JAMA 272:1851-1857

23. Shojania K, Duncan B, McDonald K, Wachter R (2001) Evidence Report/Technology Assessment $\mathrm{N}^{\circ} 43$; AHRQ publication 01E058, Making health care safer: a critical analysis of patient safety practices. Agency for Healthcare Research and Quality, Rockville

24. Watanabe R, Gilbreath K, Sakamoto C (1994) The ability of the geriatric population to read labels on over-the-counter medication containers. J Am Optom Assoc 65:32-37

25. Lambert B, Chang K, Lin S (2001) Effect of orthographic and phonological similarity on false recognition of drug names. Soc Sci Med 52:1843-1857

26. Wogalter MS, Vigilante WJ Jr (2003) Effects of label format on knowledge acquisition and perceived readability by younger and older adults. Ergonomics 46:327-344

27. Filik R, Purdy K, Gale A, Gerrett D (2004) Drug name confusion: evaluating the effectiveness of capital ("Tall Man") letters using eye movement data. Soc Sci Med 59:2597-2601

28. Buchanan T, Barker K, Gibson J, Jiang B, Pearson R (1991) Illumination and errors in dispensing. Am J Hosp Pharm 48:21372145

29. Flynn E, Barker K, Gibson J, Pearson R, Smith L, Berger B (1996) Relationships between ambient sounds and the accuracy of pharmacists' prescription-filling performance. Hum Factors 38:614-622

30. Flynn E, Barker K, Gibson J, Pearson R, Berger B, Smith L (1999) Impact of interruptions and distractions on dispensing errors in an ambulatory care pharmacy. Am J Health Syst Pharm $56: 1319-1325$

31. Bates D (2000) Using information technology to reduce rates of medication errors in hospitals. BMJ 320:788-791

32. Trunkey DD, Botney R (2001) Assessing competency: a tale of two professions. J Am Coll Surg 192:385-395

33. Amalberti R, Auroy Y, Berwick D, Barach P (2005) Five system barriers to achieving ultrasafe health care. Ann Intern Med 142:756-764 